\title{
A Brief Study of Certain Class of Harmonic Functions of Bazilevič Type
}

\author{
A. T. Oladipo ${ }^{1}$ and D. Breaz ${ }^{2}$ \\ ${ }^{1}$ Department of Pure and Applied Mathematics, Ladoke Akintola University of Technology, \\ PMB 4000, Ogbomosho, Nigeria \\ ${ }^{2}$ Department of Mathematics and Informatics, 1 Decembrie 1918 University of Alba Iulia, 5 Gabriel Bethlen Street, \\ 510009 Alba Iulia, Romania
}

Correspondence should be addressed to A. T. Oladipo; atlab_3@yahoo.com

Received 24 March 2013; Accepted 18 April 2013

Academic Editors: R. Avery, Y. Han, G. L. Karakostas, and C. Zhu

Copyright (c) 2013 A. T. Oladipo and D. Breaz. This is an open access article distributed under the Creative Commons Attribution License, which permits unrestricted use, distribution, and reproduction in any medium, provided the original work is properly cited.

We define and investigate a new subclass of Bazilevič type harmonic univalent functions using a linear operator. We investigated the harmonic structures in terms of its coefficient conditions, extreme points, distortion bounds, convolution, and convex combination. So, also, we discussed the subordination properties for the functions in this class.

\section{Introduction}

Let $A$ denote the usual class of analytic functions of the form

$$
f(z)=z+\sum_{k=2}^{\infty} a_{k} z^{k}
$$

which are analytic in the unit disk $U=\{z:|z|<1\}$ and normalized with $f(0)=0$ and $f^{\prime}(0)-1=0$. Also, we denote the subclass of $A$ consisting of analytic and univalent functions $f(z)$ in the unit disk $U$ by $S$.

Here, we recall some definitions and concepts of classes of analytic functions. Let $f \in A$. Then, $f \in S^{*}(\mu)$ if and only if

$$
\operatorname{Re}\left\{\frac{z f^{\prime}(z)}{f(z)}\right\}>\mu, \quad(0 \leq \mu<1, z \in U) .
$$

This class is called starlike class of analytic function.

Also, let $f \in A$. Then, $f \in C(\mu)$ if and only if

$$
\operatorname{Re}\left\{1+\frac{z f^{\prime \prime}(z)}{f^{\prime}(z)}\right\}>\mu, \quad(0 \leq \mu<1, z \in U) .
$$

This class is called convex class of analytic function. The above two classes have been repeatedly investigated by various authors like [1-4] just to mention but few, as the literatures littered everywhere.

The theory of analytic functions has wide application in many physical problem: problems as in heat conduction, electrostatic potential and fluid flows, and theory of fractals constitute practical examples. The concern of this work is the study of a particular family of analytic functions defined in a given domain by certain geometric conditions which are useful in the above problems.

Let $\gamma: C^{2} \rightarrow C$, and let $\phi$ be univalent in $U$. If $p$ is analytic in $U$ and satisfies the differential subordination $\Phi\left(p(z), z p^{\prime}(z)\right) \prec \phi(z)$, then $p$ is called a solution of the differential subordination. The univalent function $q$ is called a dominant of the solution of the differential subordination, $p \prec q$. If $p$ and $\Phi\left(p(z), z p^{\prime}(z)\right)$ are univalent in $U$ and satisfy the differential superordination $\phi(z) \prec \Phi\left(p(z), z p^{\prime}(z)\right)$, then $p$ is called a solution of the differential superordination. An analytic function $q$ is called subordinate of the solution of the differential superordination if $q \prec p$. For details (see [5-7]). 

tor:

Sălăgean [8] introduced the following differential opera-

$$
\begin{gathered}
D^{0} f(z)=z f^{\prime}(z), \\
D^{1} f(z)=D f(z)=z f^{\prime}(z), \\
D^{n} f(z)=D\left(D^{n-1} f(z)\right)=z\left(D^{n-1} f(z)\right)^{\prime} .
\end{gathered}
$$

From (1), we can write that

$$
f(z)^{\alpha}=\left(z+\sum_{k=2}^{\infty} a_{k} z^{k}\right)^{\alpha}
$$

Using binomial expansion on (5), we have

$$
\begin{aligned}
& f(z)^{\alpha} \\
& \quad=z^{\alpha}+\alpha a_{2} z^{\alpha+1}+\left[\alpha a_{3}+\frac{\alpha(\alpha-1)}{2 !} a_{2}^{3}\right] z^{\alpha+2} \\
& \quad+\left[\alpha a_{4}+\frac{\alpha(\alpha-1)}{2 !} 2 a_{2} a_{3}+\frac{\alpha(\alpha-1)(\alpha-2)}{3 !} a_{2}^{3}\right] z^{\alpha+3}+\cdots .
\end{aligned}
$$

We then define the class of analytic functions of fractional power $A_{\alpha}$ as

$$
f(z)^{\alpha}=z^{\alpha}+\sum_{k=\alpha}^{\infty} a_{k}(\alpha) z^{\alpha+k-1}
$$

where $\alpha>0$ (is real, and it is principal determination only).

Thus, we obtain the differential operator

$$
D^{n} f(z)^{\alpha}=\alpha^{n} z^{\alpha}+\sum_{k=\alpha}^{\infty}(\alpha+k-1)^{n} a_{k}(\alpha) z^{\alpha+k-1}
$$

Let us also define the function $\varphi_{\alpha}(a, c, z)$ by

$$
\varphi_{\alpha}(a, c ; z)=z^{\alpha}+\sum_{k=2}^{\infty} \frac{(a)_{k-1}}{c_{k-1}} z^{\alpha+k-1},
$$

$$
\left(z \in U, \alpha \in R^{+}, a \in R, c \in R-\{0,-1,-2, \ldots\}\right),
$$

where $\left(a_{k}\right)$ is the Pochhammer symbol defined by

$$
\begin{aligned}
(a)_{k} & =\frac{\gamma(a+k)}{\gamma(a)} \\
& = \begin{cases}1, & k=0, \\
a(a+1)(a+2) \cdots(a+n-1) & n \in N .\end{cases}
\end{aligned}
$$

Corresponding to the function $\varphi_{\alpha}(a, c ; z)$, we defined a linear operator

$$
J_{n}^{\alpha}(a, c) f(z)^{\alpha}=\varphi_{\alpha}(a, c ; z) * D^{n} f(z)^{\alpha}, \quad f(z)^{\alpha} \in A_{\alpha} .
$$

Or equivalently

$$
\begin{aligned}
J_{n}^{\alpha} & (a, c) f(z)^{\alpha} \\
& =\alpha^{n} z^{\alpha}+\sum_{k=2}^{\infty} \frac{(a)_{k-1}}{(c)_{k-1}}(\alpha+k-1)^{n} a_{k}(\alpha) z^{\alpha+k-1} .
\end{aligned}
$$

Remark 1. For $n=0, \alpha=1$ operator (11) reduces to Carlson-shaffer operator [3], and also for different values of $\alpha$, it imposes the Saitoh operator [7] and recently the MahzoonLotha [9]. for $a=c, \alpha=1$ poses the Salagean derivative operator.

Now, let $B_{n}^{\alpha}(\gamma)$ be the class of functions containing the operator (11) and satisfying the relation

$$
\frac{z\left(J_{n}^{\alpha}(a, c) f(z)^{\alpha}\right)^{\prime}}{\alpha J_{n}^{\alpha}(a, c) f(z)^{\alpha}} \prec\left(\frac{1+\gamma z}{1-z}\right)^{\alpha} \quad \alpha>0, \gamma \neq-1 .
$$

For $a=c, \alpha=1, \gamma=1$, and $n=0$, we obtain the well-known subclass

$$
f^{\prime}(z) \prec \frac{1+z}{1-z}
$$

Also, for $n=0, a=c$, and $\gamma=1$, we have

$$
\frac{\alpha z f^{\prime}(z)}{f(z)} \cdot \frac{f(z)^{\alpha}}{z^{\alpha}} \prec\left(\frac{1+z}{1-z}\right)^{\alpha} .
$$

For $n=0, \alpha=1$, and $\gamma=1$ we have the following subclass which contain Carlson-Shaffer operator:

$$
\frac{z(J(a, c) f(z))^{\prime}}{z} \prec \frac{1+z}{1-z} .
$$

The starting point in the study of functions defined in (13) is the discovery in 1995 by Russian Mathematician Bazilevič [10] of functions in $U$ defined by

$$
\begin{aligned}
& f(z) \\
& =\left\{\frac{\alpha}{1+\varepsilon^{2}} \int_{0}^{z} \frac{p(v)-i \varepsilon}{V\left(1+\left(i \alpha \varepsilon /\left(1+\varepsilon^{2}\right)\right)\right)} g(v)^{\alpha /\left(1+\varepsilon^{2}\right)} d v\right\}^{(1+i \varepsilon) / \alpha},
\end{aligned}
$$

where $p \in P$ and $g \in S^{*}$. The number $\alpha>0$ and $\varepsilon$ are real, and all powers are meant as principal determinant only. The family of functions in (17) became known as Bazilevic functions and is, in this work, denoted by $B(\alpha, \varepsilon)$. Except that, Bazilevič showed that each function $f \in B(\alpha, \varepsilon)$ is univalent in $U$, very little is known regarding the family as a whole. However, with some simplifications, it may be possible to understand and investigate the family. Indeed, it is easy to verify that, with special choices of the parameters $\alpha$ and $\varepsilon$ and the function $g(z)$, the family $B(\alpha, \varepsilon)$ cracks down to some well-known subclasses of univalent functions.

For instance, if we take $\varepsilon=0$, we have

$$
f(z)=\left\{\alpha \int_{0}^{z} \frac{p(v)}{v} g(v)^{\alpha} d v\right\}^{1 / \alpha} .
$$

On differentiation, the expression (18) yields

$$
\frac{z f^{\prime}(z) f(z)^{\alpha-1}}{g(z)^{\alpha}}=p(z), \quad z \in U .
$$


Or equivalently

$$
\operatorname{Re} \frac{z f^{\prime}(z) f(z)^{\alpha-1}}{g(z)^{\alpha}}>0, \quad z \in U .
$$

The subclasses of Bazilevič functions satisfying (19) are called Bazilevič functions of type $\alpha$ and are denoted by $B(\alpha)$ (see [11]). In 1973, Noonan [12] gave a plausible description of functions of the class $B(\alpha)$ as those functions in $S$ for which each $r<1$, and the tangent to the curve $U_{\alpha}(r)=$ $\left\{\varepsilon f\left(r \exp ^{i \theta}\right)^{\alpha}, 0 \leq \theta<2 \pi\right\}$ never turns back on itself as much as $\pi$ radian. If $\alpha=1$, the class $B(\alpha)$ reduces to the family of close-to-convex functions; that is,

$$
\operatorname{Re} \frac{z f^{\prime}(z)}{g(z)}>0, \quad z \in U
$$

If we decide to choose $g(z)=f(z)$ in (21), we have

$$
\operatorname{Re} \frac{z f^{\prime}(z)}{f(z)}>0, \quad z \in U,
$$

which implies that $f(z)$ is starlike. Furthermore, if we replace $f(z)$ by $z f^{\prime}(z)$ in $(22)$, we obtain

$$
\operatorname{Re}\left\{1+\frac{z f^{\prime \prime}(z)}{f^{\prime}(z)}\right\}>0, \quad z \in U
$$

which shows that $f(z)$ is convex. Moreover, if $g(z)=z$ in (20), then we have the family $B_{1}(\alpha)$ [11] of functions satisfying

$$
\operatorname{Re} \frac{z f^{\prime}(z) f(z)^{\alpha-1}}{z^{\alpha}}>0, \quad z \in U
$$

The various subfamilies of Bazilevič functions are being studied repeatedly by many authors; the literatures in this direction littered everywhere (see Bernard's Bibliography of Schlich functions [13]).

In 1992, Abduhalim [14] introduced a generalization of functions satisfying (24) as

$$
\operatorname{Re} \frac{D^{n} f(z)^{\alpha}}{z^{\alpha}}>0, \quad z \in U
$$

where the parameter $\alpha$ and the operator $D^{n}$ are defined as earlier. He denoted this class of functions by $B_{n}(\alpha)$. It is easily seen that his generalization has extraneously included analytic functions satisfying

$$
\operatorname{Re} \frac{f(z)^{\alpha}}{z^{\alpha}}>0, \quad z \in U
$$

which are largely nonunivalent in the unit disk. By proving the inclusion

$$
B_{n+1}(\alpha) \subset B_{n}(\alpha),
$$

Abdulhalim was able to show that for all $n \in N$, each function of the class $B_{n}(\alpha)$ is univalent in $U$.

Notable contributors like MacGregor, [15, 16], Noonan [12], Singh [11], Thomas [17], Tuan and Anh [18], Yamaguchi
[19], and Opoola [20] had earlier considered various special cases of the parameters $n$ and $\alpha$ of (25) and established many interesting properties of function in those particular cases.

In some general sense, it is possible to further improve work on the function defined by the geometric condition (25). Therefore, we intend to investigate this family from the viewpoints of subordination and harmonic univalent functions and determine coefficient inequalities, extreme points, distortion bounds, convolution, and convex combination.

\section{Subordination Results}

The objective of this section is to find the sufficient conditions of functions belonging to the class $B_{n}^{\alpha}(\gamma, a, c)$.

For this purpose, the following Lemmas will be necessary.

Lemma 2 (see [21]). Let $\beta \neq 0$ be a complex number. Let $q(z)$ $(q(z) \neq 0)$ be a univalent function in $U$ such that

$$
\operatorname{Re}\left\{1+\frac{z q^{\prime \prime}(z)}{q^{\prime}(z)}-\frac{z q^{\prime}(z)}{q(z)}\right\}>\max \left\{0, R\left(\frac{\beta-1}{\beta} q(z)\right)\right\} .
$$

If $p,(p(z) \neq 0), z \in U$ satisfies the differential subordination

$$
\begin{aligned}
(1-\beta) & (p(z)-1)+\beta \frac{z p^{\prime}(z)}{p(z)} \\
& \prec(1-\beta)(q(z)-1)+\beta \frac{z q^{\prime}(z)}{q(z)},
\end{aligned}
$$

then $p \prec q$ and $q$ is the best dominant.

Lemma 3 (see [22]). Let $\omega$ be analytic in $U$ with $\omega(0)=0$. If $|\omega(z)|$ attains its maximum value on the circle $|z|<1$ at a point $z_{0}$, then

$$
z_{0} \omega^{\prime}\left(z_{0}\right)=m \omega\left(z_{0}\right),
$$

where $m$ is a real number and $m \geq 1$.

Lemma 4 (see [23]). If $f \in A$ satisfies

$$
\operatorname{Re}\left[1+\frac{z f^{\prime \prime}(z)}{f^{\prime}(z)}\right]<\frac{(\mu+1)}{2(\mu-1)}, \quad(2 \leq \mu<3, z \in U),
$$

then

$$
\begin{gathered}
\frac{z f^{\prime}(z)}{f(z)} \prec \frac{\mu(1-z)}{\mu-z}, \\
\left|\frac{z f^{\prime}(z)}{f(z)}-\frac{\mu}{\mu+1}\right|<\frac{\mu}{\mu+1} .
\end{gathered}
$$

Now, we begin our main results as the following.

Theorem 5. Let $\alpha>0$ ( $\alpha$ is real), $n \in N_{0}$, and $\beta \neq 0$ be a complex number such that

$$
\begin{aligned}
& \operatorname{Re}\left\{1+\frac{z(1-\gamma+2 \gamma z)}{(1+\gamma z)(1-z)}\right\} \\
& >\max \left\{0, R\left(\frac{\beta-1}{\beta}\right)\left(\frac{1+\gamma z}{1-z}\right)^{\alpha}\right\} .
\end{aligned}
$$


If the subordination

$$
\begin{aligned}
(1-\beta) & {\left[\frac{z\left(J_{n}^{\alpha}(a, c) f(z)^{\alpha}\right)^{\prime}}{\alpha^{n} z^{\alpha}}-1\right] } \\
& +\beta\left(1-\alpha+\frac{z\left(J_{n}^{\alpha}(a, c) f(z)^{\alpha}\right)^{\prime \prime}}{\left(J_{n}^{\alpha}(a, c) f(z)^{\alpha}\right)^{\prime}}\right) \\
< & (1-\beta)\left[\left(\frac{1+\gamma z}{1-z}\right)^{\alpha}-1\right]+\frac{\alpha \beta(1+\gamma) z}{(1+\gamma z)(1-z)}
\end{aligned}
$$

holds, then $f^{\alpha} \in B_{n}^{\alpha}(\gamma, a, c)$.

Proof. Suppose that

$$
p(z)=\frac{z\left(J_{n}^{\alpha}(a, c) f(z)^{\alpha}\right)^{\prime}}{\alpha^{n} z^{\alpha}}, \quad q(z)=\left(\frac{1+\gamma z}{1-z}\right)^{\alpha} .
$$

Then, simple computations give

$$
\begin{aligned}
\operatorname{Re}\{1 & \left.+\frac{z q^{\prime \prime}(z)}{q^{\prime}(z)}-\frac{z q^{\prime}(z)}{q(z)}\right\} \\
= & \operatorname{Re}\left\{1+\frac{z(1-\gamma+2 \gamma z)}{(1+\gamma z)(1-z)}\right\} \\
> & \max \left\{0, R\left(\frac{\beta-1}{\beta}\right)\left(\frac{1+\gamma z}{1-z}\right)^{\alpha}\right\} \\
= & \max \left\{0, R\left(\frac{\beta-1}{\beta}\right) q(z)\right\}, \\
(1-\beta) & (p(z)-1)+\beta \frac{z p^{\prime}(z)}{p(z)} \\
= & (1-\beta)\left[\frac{z\left(J_{n}^{\alpha}(a, c) f(z)^{\alpha}\right)^{\prime}}{\alpha^{n} z^{\alpha}}-1\right] \\
& +\beta\left(1-\alpha+\frac{z\left(J_{n}^{\alpha}(a, c) f(z)^{\alpha}\right)^{\prime \prime}}{\left(J_{n}^{\alpha}(a, c) f(z)^{\alpha}\right)^{\prime}}\right) \\
& <(1-\beta)\left[\left(\frac{1+\gamma z}{1-z}\right)^{\alpha}-1\right]+\frac{\alpha \beta(1+\gamma) z}{(1+\gamma z)(1-z)} \\
= & (1-\beta)(q(z)-1)+\beta \frac{z q^{\prime}(z)}{q(z)} .
\end{aligned}
$$

Thus, in the view of Lemma 2, we have $f(z)^{\alpha} \in B_{n}^{\alpha}(\gamma, a, c)$.

For $\alpha=1, \gamma=1, n=0$. We have the following.

Corollary 6. Let $\beta \neq 0$ be a complex number such that

$$
\operatorname{Re}\left\{1+\frac{2 z^{2}}{1-z^{2}}\right\}>\max \left\{0, \operatorname{Re}\left(\frac{\beta-1}{\beta}\left(\frac{1+z}{1-z}\right)\right)\right\} .
$$

If the subordination

$$
\begin{aligned}
(1-\beta) & {\left[\left(J_{0}^{1}(a, c) f(z)\right)^{\prime}-1\right]+\beta\left(\frac{z\left(J_{0}^{1}(a, c) f(z)\right)^{\prime \prime}}{\left(J_{0}^{1}(a, c) f(z)\right)^{\prime}}\right) } \\
\prec & (1-\beta)\left[\left(\frac{1+z}{1-z}\right)-1\right]+\frac{2 \beta z}{1-z^{2}}
\end{aligned}
$$

Holds, then $f(z)^{\alpha} \in B_{0}^{1}(1, a, c)$.

$$
\text { For } a=c, \alpha=1, \gamma=1 \text {, and } n=0 \text {, we have the following }
$$

Corollary 7. Let $\beta \neq 0$ be a complex number such that

$$
\operatorname{Re}\left\{1+\frac{2 z^{2}}{1-z^{2}}>\max \left\{0, R\left(\frac{\beta-1}{\beta}\left(\frac{1+z}{1-z}\right)\right)\right\} .\right.
$$

If the subordination

$$
\begin{aligned}
(1- & \beta)\left(f^{\prime}(z)-1\right)+\beta \frac{z f^{\prime \prime}(z)}{f^{\prime}(z)} \\
& <(1-\beta)\left[\frac{1+z}{1-z}-1\right]+\frac{2 \beta z}{1-z^{2}}
\end{aligned}
$$

holds, then $f(z)^{\alpha} \in B_{0}^{1}(1,1,1) \equiv B$.

For $\gamma=1, a=c$, we have the following.

Corollary 8. Let $\beta \neq 0$ be a complex number such that

$$
\operatorname{Re}\left\{1+\frac{2 z^{2}}{1-z^{2}}\right\}>\max \left\{0, \operatorname{Re}\left(\frac{\beta-1}{\beta}\left(\frac{1+z}{1-z}\right)^{\alpha}\right)\right\} .
$$

If the subordination

$$
\begin{aligned}
(1 & -z)\left[\frac{z\left(J_{n}^{\alpha} f(z)^{\alpha}\right)^{\prime}}{\alpha^{n} z^{\alpha}}-1\right]+\beta\left(1-\alpha+\frac{z\left(J_{n}^{\alpha} f(z)^{\alpha}\right)^{\prime \prime}}{\left(J_{n}^{\alpha} f(z)^{\alpha}\right)^{\prime}}\right) \\
& <(1-\beta)\left[\left(\frac{1+z}{1-z}\right)^{\alpha}-1\right]+\frac{2 \alpha \beta z}{1-z^{2}}
\end{aligned}
$$

holds, then $f^{\alpha} \in B_{n}^{\alpha}(1,1,1)$.

For $\gamma=1, n=0, a=c$, we have the following.

Corollary 9. Let $\beta \neq 0$ be a complex number such that

$$
\operatorname{Re}\left\{1+\frac{2 z^{2}}{1-z^{2}}\right\}>\max \left\{0, \operatorname{Re}\left(\frac{\beta-1}{\beta}\left(\frac{1+z}{1-z}\right)^{\alpha}\right)\right\} .
$$

If the subordination

$$
\begin{aligned}
&(1-\beta)\left[\frac{\alpha z f^{\prime}(z)}{f(z)} \cdot \frac{f(z)^{\alpha}}{z^{\alpha}}-1\right]+\beta\left(1-\beta+\frac{z\left(f(z)^{\alpha}\right)^{\prime \prime}}{\left(f(z)^{\alpha}\right)^{\prime}}\right) \\
&<(1-\beta)\left[\left(\frac{1+z}{1-z}\right)^{\alpha}-1\right]+\frac{2 \alpha \beta z}{1-z^{2}}
\end{aligned}
$$

holds, then $f^{\alpha} \in B_{0}^{\alpha} \equiv B(\alpha)$. 
Theorem 10. Let the functions $f(z)^{\alpha}$ take the form (7) and satisfy

$$
\begin{aligned}
\operatorname{Re}\{1 & \left.-\alpha+\frac{z\left(J_{n}^{\alpha}(a, c) f(z)^{\alpha}\right)^{\prime \prime}}{\left(J_{n}^{\alpha}(a, c) f(z)^{\alpha}\right)^{\prime}}\right\} \\
& <\frac{\alpha(\gamma+1)}{2(\gamma-1)}, \quad z \in U, \alpha>0, \gamma>1 .
\end{aligned}
$$

Then, $f \in B_{n}^{\alpha}(\gamma, a, c)$.

Proof. Let $\omega$ be defined by

$$
\frac{z\left(J_{n}^{\alpha}(a, c) f(z)^{\alpha}\right)^{\prime}}{\alpha^{n} z^{\alpha}}=\left(\frac{1+\gamma \omega(z)}{1-\omega(z)}\right)^{\alpha}, \quad \omega(z) \neq 1 .
$$

Then, $\omega$ is analytic in $U$, and since $\gamma \neq-1$, then $\omega(0)=0$. Also, it follows that

$$
\begin{aligned}
\operatorname{Re}\{1 & \left.-\alpha+\frac{z\left(J_{n}^{\alpha}(a, c) f(z)^{\alpha}\right)^{\prime \prime}}{\left(J_{n}^{\alpha}(a, c) f(z)^{\alpha}\right)^{\prime}}\right\} \\
& =\operatorname{Re}\left\{\frac{\alpha(\gamma+1) z \omega^{\prime}(z)}{(1+\gamma \omega(z))(1-\omega(z))}\right\} \\
& <\frac{\alpha(\gamma+1)}{2(\gamma-1)}, \quad \gamma \neq 1 .
\end{aligned}
$$

Now, let us proceed to prove that $|\omega(z)|<1$. Suppose that there exists a point $z_{0} \in U$ such that

$$
\max _{|z| \leq\left|z_{0}\right|}|\omega(z)|=|\omega(z)|=1
$$

Then, using Lemma 3 and letting $\omega\left(z_{0}\right)=e^{i \theta}$ and $z_{0} \omega^{\prime}\left(z_{0}\right)=$ $m e^{i \theta}, m \geq 1$ yields that

$$
\begin{aligned}
\operatorname{Re} & \left\{1-\alpha+\frac{z\left(J_{n}^{\alpha}(a, c) f(z)^{\alpha}\right)^{\prime \prime}}{\left(J_{n}^{\alpha}(a, c) f(z)^{\alpha}\right)^{\prime}}\right\} \\
& =\operatorname{Re}\left\{\frac{\alpha(\gamma+1) z_{0} \omega^{\prime}\left(z_{0}\right)}{\left(1+\gamma \omega\left(z_{0}\right)\right)\left(1-\omega\left(z_{0}\right)\right)}\right\} \\
& =\operatorname{Re}\left\{\frac{\alpha m e^{i \theta}(\gamma+1)}{\left(1+\gamma \omega\left(e^{i \theta}\right)\right)\left(1-e^{i \theta}\right)}\right\} \\
& \left.=\frac{\alpha m(1+\gamma)}{2(\gamma-1)} \geq \frac{\alpha(\gamma+1)}{2(\gamma-1)}, \quad \gamma>1, \alpha>0 \text { ( } \alpha \text { is real }\right) .
\end{aligned}
$$

Thus, we have

$$
\operatorname{Re}\left\{1-\alpha+\frac{z\left(J_{n}^{\alpha}(a, c) f(z)^{\alpha}\right)^{\prime \prime}}{\left(J_{n}^{\alpha}(a, c) f(z)^{\alpha}\right)^{\prime}}\right\} \geq \frac{\alpha(\gamma+1)}{2(\gamma-1)}, \quad z \in U,
$$

which contradicts the hypothesis (46). Therefore, we conclude that $|\omega(z)|<1$ for all $z \in U$ and

$$
\begin{gathered}
\frac{z\left(J_{n}^{\alpha}(a, c) f(z)^{\alpha}\right)^{\prime}}{\alpha^{n} z^{\alpha}} \\
z \in\left(\frac{1+\gamma(z)}{1-(z)}\right)^{\alpha}, \\
z \in U, \quad \gamma \neq-1, \quad \alpha>0,
\end{gathered}
$$

where ( $\alpha$ is real). This completes the proof of the theorem.

Letting $\alpha=1, n=0$, and $a=c$ in Theorem 10, we have the following.

Corollary 11. Let the function $f$ take the form (1) and satisfy

$$
\operatorname{Re}\left\{\frac{z f^{\prime \prime}(z)}{f^{\prime}(z)}\right\}<\frac{\gamma+1}{2(\gamma-1)}, \quad z \in U, \gamma>1
$$

Then,

$$
f^{\prime}(z) \prec \frac{1+\gamma z}{1-z}
$$

Corollary 12. Let the function $f$ take the form (1) and satisfy

$$
\operatorname{Re}\left\{\frac{z f^{\prime \prime}}{f^{\prime}(z)}\right\}<\frac{\gamma+1}{2(\gamma-1)}, \quad(z \in U), \gamma>1
$$

Then,

$$
\left|f^{\prime}(z)-\frac{\gamma}{2}\right|<\frac{\gamma}{2}, \quad(z \in U)
$$

With various special choices of the parameters involved, many existing and new subclasses of Bazilevič functions could be derived.

\section{Harmonic Structure of Bazilevič Type}

In this section, the authors wish to have a look into the Bazilevič type harmonic univalent functions.

A continuous complex-valued function $f=u+i v$ defined in a simply connected domain $D$ is said to be harmonic in $D$. In any simply connected domain, we can write

$$
f=h+\bar{g}
$$

where $h$ and $g$ are analytic in $D$. We call $h$ the analytic part and $g$ the coanalytic part of $A$. A necessary and sufficient condition for $f$ to be locally univalent and sense preserving in $D$ is that

$$
\left|h^{\prime}(z)\right|>\left|g^{\prime}(z)\right|, \quad(z \in U) .
$$

Denote by $S_{H}$ the class of functions $f$ of the form (57) that are harmonic univalent and sense-preserving in the disk $U$. The subclasses of harmonic functions have been studied by some authors for different purposes with different properties (see [24-26]). But unfortunately, it is becoming very difficult to see the literatures on Bazilevič-type harmonic univalent function, and this may be likely associated with the problem 
index $\alpha$ always poses. This paper is designed to address this issue.

In this work, we may express the analytic functions $h$ and $g$ as

$$
\begin{gathered}
h(z)^{\alpha}=z^{\alpha}+\sum_{k=2}^{\infty} a_{k}(\alpha) z^{\alpha+k-1}, \\
g(z)^{\alpha}=\sum_{k=1}^{\infty} b_{k}(\alpha) z^{\alpha+k-1}, \quad\left|b_{1}(\alpha)\right|<1 .
\end{gathered}
$$

Therefore,

$$
f(z)^{\alpha}=h(z)^{\alpha}+\overline{g(z)^{\alpha}} .
$$

We define our linear operator as given in (11) such that

$$
J_{n}^{\alpha}(a, c) f(z)^{\alpha}=J_{n}^{\alpha}(a, c) h(z)^{\alpha}+(-1)^{n} \overrightarrow{J_{n}^{\alpha}(a, c) g(z)^{\alpha}},
$$

where

$$
\begin{gathered}
J_{n}^{\alpha}(a, c) h(z)^{\alpha}=\alpha^{n} z^{\alpha}+\sum_{k=2}^{\infty} \frac{a_{k-1}}{c_{k-1}}(\alpha+k-1)^{n} a_{k}(\alpha) z^{\alpha+k-1} \\
J_{n}^{\alpha}(a, c) g(z)^{\alpha}=\sum_{k=1}^{\infty} \frac{a_{k-1}}{c_{k-1}}(\alpha+k-1)^{n} b_{k}(\alpha) z^{\alpha+k-1}
\end{gathered}
$$

We let $B_{n}^{\alpha}(\beta, a, c)$ be the family of harmonic functions $f$ of the form (57) such that

$$
\operatorname{Re}\left\{\frac{J_{n}^{\alpha}(a, c) f(z)^{\alpha}}{\alpha^{n} z^{\alpha}}\right\}>\beta, \quad 0 \leq \beta<1,
$$

where $\alpha>0$, ( $\alpha$ is real), $n \in N_{0}$, and $J_{n}^{\alpha}(a, c)$ is earlier defined in (11).

Furthermore, let the subclass $V B_{n}^{\alpha} H(\beta, a, c)$ consist of harmonic functions

$$
f_{n}^{\alpha}=h^{\alpha}+\overline{g_{n}^{\alpha}}
$$

so that $h^{\alpha}$ and $g_{n}^{\alpha}$ are of the form

$$
\begin{gathered}
h(z)^{\alpha}=z^{\alpha}+\sum_{k=2}^{\infty}\left|a_{k}(\alpha)\right| z^{\alpha+k-1} \\
g(z)^{\alpha}=(-1)^{n}+\sum_{k=1}^{\infty}\left|b_{k}(\alpha) z^{\alpha+k-1}\right|, \quad\left|b_{1}(\alpha)\right|<1 .
\end{gathered}
$$

The authors in this work wish to study the Bazilevič-type harmonic univalent functions defined by linear operator in which $h^{\alpha}$ has positive coefficients. We claim that our results are quite new and not explored in the literatures.

Assigning specific values to $n, \beta, a, c, \alpha$ in the subclass $B_{n}^{\alpha} H(\beta, a, c)$, we obtain the following subclasses which may be the expected results by using definition of earlier authors of subclasses of Bazilevič functions such as classes studied by Abduhalim [14], Yamaguchi [19], Macgregor [15], and Singh [11], just to mention but few.

We first prove a sufficient condition for the function in $B_{n}^{\alpha} H(\beta, a, c)$.
Theorem 13. Let $f^{\alpha}=h^{\alpha}+\overline{g^{\alpha}}$, where $f^{\alpha}$ and $g^{\alpha}$ are as earlier defined if

$$
\begin{aligned}
& \sum_{k=2}^{\infty} \frac{(a)_{k-1}}{(c)_{k-1}}\left(\frac{\alpha+k-1}{\alpha}\right)^{n}\left|a_{k}(\alpha)\right| \\
& \quad+\sum_{k=1}^{\infty} \frac{(a)_{k-1}}{(c)_{k-1}}\left(\frac{\alpha+k-1}{\alpha}\right)^{n}\left|b_{k}(\alpha)\right| \leq 1-\beta,
\end{aligned}
$$

where $\in N_{0}, 0 \leq \beta<1, a \in R, c \in R-\{-1,-2, \ldots\}$ and $\alpha>0$; is real then $f(z)^{\alpha}$ is sense-preserving, harmonic univalent in $U$, and $f \in B_{n}^{\alpha} H(\beta, a, c)$.

Proof. If $z_{1}^{\alpha} \neq z_{2}^{\alpha}$, then

$$
\begin{aligned}
& \left|\frac{f\left(z_{1}\right)^{\alpha}-f\left(z_{2}\right)^{\alpha}}{h\left(z_{1}\right)^{\alpha}-h\left(z_{2}\right)^{\alpha}}\right| \\
& \geq 1-\left|\frac{g\left(z_{1}\right)^{\alpha}-g\left(z_{2}\right)^{\alpha}}{h\left(z_{1}\right)^{\alpha}-h\left(z_{2}\right)^{\alpha}}\right| \\
& =1-\left|\frac{\sum_{k=1}^{\infty} b_{k}(\alpha)\left(z_{1}^{\alpha+k-1}-z_{2}^{\alpha+k-1}\right)}{\left(z_{1}^{\alpha}-z_{2}^{\alpha}\right)+\sum_{k=2}^{\infty} a_{k}(\alpha)\left(z_{1}^{\alpha+k-1}-z_{2}^{\alpha+k-1}\right)}\right| \\
& >1-\frac{\sum_{k=1}^{\infty}(\alpha+k-1) b_{k}(\alpha)}{\alpha+\sum_{k=2}^{\infty}(\alpha+k-1) a_{k}(\alpha)} \\
& \geq 1-\left(\left(\sum_{k=1}^{\infty}\left((a)_{k-1} /(c)_{k-1}\right)((\alpha+k-1) / \alpha)^{n}\right.\right. \\
& \left.\times(1 /(1-\beta))\left|b_{k}(\alpha)\right|\right) \\
& \times\left(1+\sum_{k=2}^{\infty}\left((a)_{k-1} /(c)_{k-1}\right)((\alpha+k-1) / \alpha)^{n}\right. \\
& \left.\left.\times(1 /(1-\beta))\left|a_{k}(\alpha)\right|\right)^{-1}\right)
\end{aligned}
$$

$\geq 0$,

which proves the univalence. Note that $f$ is sense-preserving in $U$. This is because

$$
\begin{aligned}
\left|h(z)^{\alpha}\right| & \geq \alpha-\sum_{k=2}^{\infty}(\alpha+k-1)\left|a_{k}(\alpha)\right||z|^{\alpha+k-1} \\
& >\alpha-\sum_{k=2}^{\infty}(\alpha+k-1)\left|a_{k}(\alpha)\right| \\
& \geq \alpha^{n}-\sum_{k=2}^{\infty} \frac{(a)_{k-1}}{(c)_{k-1}} \frac{(\alpha+k-1)^{n}}{1-\beta}\left|a_{k}(\alpha)\right| \\
& \geq \sum_{k=1}^{\infty} \frac{(a)_{k-1}}{(c)_{k-1}} \frac{(\alpha+k-1)^{n}}{1-\beta}\left|b_{k}(\alpha)\right|
\end{aligned}
$$




$$
\begin{aligned}
& \geq \sum_{k=1}^{\infty}(\alpha+k-1)\left|b_{k}(\alpha)\right| \\
& >\sum_{k=2}^{\infty}(\alpha+k-1)\left|b_{k}(\alpha)\right|\left|z^{\alpha+k-2}\right| \\
& \geq\left|g(z)^{\alpha}\right| .
\end{aligned}
$$

By (63) and (64), we have

$$
\begin{aligned}
\operatorname{Re} & \left\{\frac{J_{n}^{\alpha}(a, c) f(z)^{\alpha}}{\alpha^{n} z^{n}}\right\} \\
& =\operatorname{Re}\left\{\frac{J_{n}^{\alpha}(a, c) h(z)^{\alpha}+(-1)^{n} \overline{J_{n}^{\alpha}(a, c) g(z)^{\alpha}}}{\alpha^{n} z^{n}}\right\}>\beta .
\end{aligned}
$$

Using the fact that $\operatorname{Re}(\omega)>\beta$ if and only if $|1-\beta+\omega| \geq$ $|1+\beta-\omega|$, it suffices to show that

$$
\left|1-\beta+\frac{J_{n}^{\alpha}(a, c) f(z)^{\alpha}}{\alpha^{n} z^{n}}\right|-\left|1-\beta-\frac{J_{n}^{\alpha}(a, c) f(z)^{\alpha}}{\alpha^{n} z^{n}}\right| \geq 0 .
$$

That is,

$$
\begin{aligned}
& \left|(1-\beta) \alpha^{n} z^{n}+J_{n}^{\alpha}(a, c) f(z)^{\alpha}\right| \\
& -\left|(1-\beta) \alpha^{n} z^{n}-J_{n}^{\alpha}(a, c) f(z)^{\alpha}\right| \geq 0, \\
& \left|(1-\beta) \alpha^{n} z^{\alpha}+J_{n}^{\alpha}(a, c) h(z)^{\alpha}+(-1)^{n} \overline{J_{n}^{\alpha}(a, c) g(z)^{\alpha}}\right| \\
& -\left|(1-\beta) \alpha^{n} z^{\alpha}-J_{n}^{\alpha}(a, c) h(z)^{\alpha}-(-1)^{n} J_{n}^{\alpha}(a, c) g(z)^{\alpha}\right| \\
& =\mid \alpha^{n} z^{\alpha}-\beta \alpha^{n} z^{\alpha}+\alpha^{n} z^{\alpha} \\
& \quad+\sum_{k=2}^{\infty} \frac{(a)_{k-1}}{(c)_{k-2}}(\alpha+k-1)^{n} a_{k}(\alpha) z^{\alpha+k-1} \\
& +(-1)^{n} \sum_{k=1}^{\infty} \frac{(a)_{k-1}}{(c)_{k-1}}(\alpha+k-1)^{n} \overline{b_{k}(\alpha) z^{\alpha+k-1}} \mid \\
& -\mid \alpha^{n} z^{\alpha}+\beta \alpha^{n} z^{\alpha}-\alpha^{n} z^{\alpha} \\
& \quad-\sum_{k=2}^{\infty} \frac{(a)_{k-1}}{(c)_{k-2}}(\alpha+k-1)^{n} a_{k}(\alpha) z^{\alpha+k-1} \\
& \quad-(-1)^{n} \sum_{k=1}^{\infty} \frac{(a)_{k-1}}{(c)_{k-1}}(\alpha+k-1)^{n} \overline{b_{k}(\alpha) z^{\alpha+k-1}} \mid, \\
& +(-1)^{n} \sum_{k=1}^{\infty} \frac{(a)_{k-1}}{(c)_{k-1}}(\alpha+k-1)^{n} b_{k}(\alpha) z^{\alpha+k-1} \mid \\
& =\mid \alpha^{n}(2-\beta) z^{\alpha}+\sum_{k=2}^{\infty} \frac{(a)_{k-1}}{(c)_{k-2}}(\alpha+k-1)^{n} a_{k}(\alpha) z^{\alpha+k-1}
\end{aligned}
$$

$$
\begin{gathered}
-\mid \beta \alpha^{n} z^{\alpha}-\sum_{k=2}^{\infty} \frac{(a)_{k-1}}{(c)_{k-2}}(\alpha+k-1)^{n} a_{k}(\alpha) z^{\alpha+k-1} \\
-(-1)^{n} \sum_{k=1}^{\infty} \frac{(a)_{k-1}}{(c)_{k-1}}(\alpha+k-1)^{n} b_{k}(\alpha) z^{\alpha+k-1} \mid \\
\geq 2 \alpha^{n}(1-\beta)|z|^{\alpha} \\
+\sum_{k=2}^{\infty} 2 \frac{(a)_{k-1}}{(c)_{k-1}}(\alpha+k-1)^{n}\left|a_{k}(\alpha)\right||z|^{\alpha+k-1} \\
-(-1)^{n} \sum_{k=1}^{\infty} 2 \frac{(a)_{k-1}}{(c)_{k-1}}(\alpha+k-1)^{n}\left|b_{k}(\alpha)\right||z|^{\alpha+k-1} \\
\times 2 \alpha^{n}(1-\beta)\left[1+\sum_{k=2}^{\infty} \frac{1}{1-\beta} \frac{(a)_{k-1}}{(c)_{k-1}}\left(\frac{\alpha+k-1}{\alpha}\right)^{n}\right. \\
\times\left|a_{k}(\alpha)\right| \\
+(-1)^{n} \sum_{k=1}^{\infty} \frac{1}{1-\beta} \frac{(a)_{k-1}}{(c)_{k-1}} \\
\left.\times\left(\frac{\alpha+k-1}{\alpha}\right)^{n}\left|b_{k}(\alpha)\right|\right] .
\end{gathered}
$$

This last expression is nonnegative by (66), and so the proof is complete.

The harmonic function

$$
\begin{aligned}
f(z)^{\alpha}= & z^{\alpha}+\sum_{k=2}^{\infty} \frac{(c)_{k-1}(1-\beta)}{(a)_{k-1}}\left(\frac{\alpha}{\alpha+k-1}\right)^{n} x_{k} z^{\alpha+k-1} \\
& +\sum_{k=1}^{\infty} \frac{(c)_{k-1}(1-\beta)}{(a)_{k-1}}\left(\frac{\alpha}{\alpha+k-1}\right)^{n} \overline{y^{\alpha+k-1}}
\end{aligned}
$$

where $n \in N_{0}, \alpha>0$ ( $\alpha$ is real), $0 \leq \beta<1, a \in R, c \in$ $R-\{-1,0,1 \ldots\}$ and

$$
\sum_{k=2}^{\infty}\left|x_{k}\right|+\sum_{k=1}^{\infty}\left|y_{k}\right|=1
$$

shows that the coefficient bound given by (66) is sharp. The functions of the form (72) are in $B_{n}^{\alpha} H(\beta, a, c)$ because

$$
\begin{aligned}
\sum_{k=2}^{\infty} & \frac{1}{1-\beta} \frac{(a)_{k-1}}{(c)_{k-1}}\left(\frac{\alpha+k-1}{\alpha}\right)^{n}\left|a_{k}(\alpha)\right| \\
& +\sum_{k=1}^{\infty} \frac{1}{1-\beta} \frac{(a)_{k-1}}{(c)_{k-1}}\left(\frac{\alpha+k-1}{\alpha}\right)^{n}\left|b_{k}(\alpha)\right| \\
& =\sum_{k=2}^{\infty}\left|x_{k}\right|+\sum_{k=1}^{\infty}\left|y_{k}\right|=1 .
\end{aligned}
$$

In the following theorem, it is shown that the condition (66) is also necessary for functions $f_{n}^{\alpha}=h^{\alpha}+g_{n}^{\alpha}$, where $h^{\alpha}$ and $g_{n}^{\alpha}$ are as earlier defined. 
Theorem 14. Let $f_{n}^{\alpha}=h^{\alpha}+\overline{g_{n}^{\alpha}}$. Then, $f_{n} \in V B_{n}^{\alpha} H(\beta, a, c)$ if and only if

$$
\begin{aligned}
\sum_{k=2}^{\infty} & \frac{1}{1-\beta} \frac{(a)_{k-1}}{(c)_{k-1}}\left(\frac{\alpha+k-1}{\alpha}\right)^{n}\left|a_{k}(\alpha)\right| \\
& +\sum_{k=1}^{\infty} \frac{1}{1-\beta} \frac{(a)_{k-1}}{(c)_{k-1}}\left(\frac{\alpha+k-1}{\alpha}\right)^{n}\left|b_{k}(\alpha)\right| \leq 1 .
\end{aligned}
$$

Proof. Since $V B_{n}^{\alpha} H(\beta, a, c) \subset B_{n}^{\alpha} H(\beta, a, c)$, we only need to prove the "only if" part of the theorem. To this end, for functions $f_{n}^{\alpha}$ of the form (64), we notice that the condition

$$
\operatorname{Re}\left\{\frac{J_{n}^{\alpha}(a, c) f_{n}(z)^{\alpha}}{\alpha^{n} z^{\alpha}}\right\}>\beta
$$

is equivalent to

$$
\begin{aligned}
\operatorname{Re}\{( & (1-\beta) z^{\alpha}-\sum_{k=2}^{\infty} \frac{(a)_{k-1}}{(c)_{k-1}}\left(\frac{\alpha+k-1}{\alpha}\right)^{n} a_{k}(\alpha) z^{\alpha+k-1} \\
& \left.\left.-(-1)^{2 n} \sum_{k=1}^{\infty} \frac{(a)_{k-1}}{(c)_{k-1}}\left(\frac{\alpha+k-1}{\alpha}\right)^{n} b_{k}(\alpha) z^{\alpha+k-1}\right)\left(z^{-\alpha}\right)\right\}
\end{aligned}
$$

$\geq 0$.

The above required condition (75) must hold for all values of $z$ in $U$. Upon clearing the values of $z$ on the positive real axis, where $0 \leq z=r<1$, we must have

$$
\begin{aligned}
& (1-\beta) z^{\alpha}-\sum_{k=2}^{\infty} \frac{(a)_{k-1}}{(c)_{k-1}}\left(\frac{\alpha+k-1}{\alpha}\right)^{n} a_{k}(\alpha) r^{k-1} \\
& -(-1)^{n} \sum_{k=1}^{\infty} \frac{(a)_{k-1}}{(c)_{k-1}}\left(\frac{\alpha+k-1}{\alpha}\right)^{n} b_{k}(\alpha) r^{k-1} \geq 0,
\end{aligned}
$$

and the proof is complete.

Theorem 15. Let $f_{n}^{\alpha}=h^{\alpha}+\overline{g^{\alpha}}$, where $h^{\alpha}$ and $g^{\alpha}$ are as given earlier. Then, $f_{n} \in V B_{n}^{\alpha} H(\beta, a, c)$ if and only if

$$
f_{n}(z)^{\alpha}=\sum_{k=1}^{\infty}\left(x_{k} h_{k}(z)+y_{k} g_{n k}(z)\right),
$$

where $h_{1}(z)^{\alpha}=z^{\alpha}, h_{k}(z)^{\alpha}=z^{\alpha}+\left((c)_{k-1}(1-\beta)(\alpha)^{n} /(a)_{k-1}(\alpha+\right.$ $\left.k-1)^{n}\right) z^{\alpha+k-1}(k=2,3, \ldots)$,

$$
\begin{aligned}
& g_{m k}(z)^{\alpha} \\
& \quad=z^{\alpha}+(-1)^{n} \frac{\alpha^{n}(1-\beta)(c)_{k-1}}{(\alpha+k-1)^{n}(a)_{k-1}} \overline{z^{\alpha+k-1}} \quad(k=2,3, \ldots),
\end{aligned}
$$

where $x_{k} \geq 0, y_{k} \geq 0, x_{1}=1-\left(\sum_{k=2}^{\infty} x_{k}+\sum_{k=1}^{\infty} y_{k}\right) \geq 0$.

In particular, the extreme points of $V B_{n}^{\alpha} H\left(\beta, a, c\right.$ are $\left\{b_{k}\right\}$ and $\left.\left\{g_{n k}\right\}\right)$.
Proof. For functions $f^{\alpha}=h^{\alpha}+\overline{g^{\alpha}}$, where $h^{\alpha}$ and $g^{\alpha}$ are as earlier defined, we have

$$
\begin{aligned}
f_{n}(z)^{\alpha}= & \sum_{k=1}^{\infty}\left(x_{k} h_{k}(z)+y_{k} g_{m k}(z)\right) \\
= & \sum_{k=1}^{\infty}\left(x_{k}+y_{k}\right) z^{\alpha}+\sum_{k=2}^{\infty} \frac{\alpha^{n}(1-\beta)(c)_{k-1}}{(\alpha+k-1)^{n}(a)_{k-1}} x_{k} z^{\alpha+k-1} \\
& +(-1)^{n} \sum_{k=1}^{\infty} \frac{\alpha^{n}(1-\beta)(c)_{k-1}}{(\alpha+k-1)^{n}(a)_{k-1}} y_{k} z^{\alpha+k-1} .
\end{aligned}
$$

Then,

$$
\begin{aligned}
& \sum_{k=2}^{\infty} \frac{(a)_{k-1}}{(c)_{k-1}}\left(\frac{\alpha+k-1}{\alpha}\right)^{n} \frac{1}{1-\beta}\left|a_{k}(\alpha)\right| \\
& \quad+\sum_{k=1}^{\infty} \frac{(a)_{k-1}}{(c)_{k-1}}\left(\frac{\alpha+k-1}{\alpha}\right)^{n} \frac{1}{1-\beta}\left|b_{k}(\alpha)\right| \\
& =\sum_{k=2}^{\infty} x_{k}+\sum_{k=2}^{\infty} x_{k}=1-x_{1} \leq 1,
\end{aligned}
$$

and so $f_{n}^{\alpha} \in B_{n}^{\alpha} H(\beta, a, c)$.

Conversely, suppose that $f_{n}^{\alpha} \in B_{n}^{\alpha} H(\beta, a, c)$. Setting

$$
\begin{array}{r}
x_{k}=\frac{1}{1-\beta} \frac{(a)_{k-1}}{(c)_{k-1}}\left(\frac{\alpha+k-1}{\alpha}\right)^{n}\left|a_{k}(\alpha)\right|, \\
0 \leq x_{k} \leq 1 \quad(k=2,3, \ldots), \\
y_{k}=\frac{1}{1-\beta} \frac{(a)_{k-1}}{(c)_{k-1}}\left(\frac{\alpha+k-1}{\alpha}\right)^{n}\left|b_{k}(\alpha)\right|, \\
0 \leq y_{k} \leq 1 \quad(k=1,2,3, \ldots),
\end{array}
$$

and $x_{1}=1-\sum_{k=2}^{\infty} x_{k}-\sum_{k=1}^{\infty} y_{k}$, therefore, $f_{n}^{\alpha}$ can be written as

$$
\begin{aligned}
f_{n}(z)^{\alpha}= & z^{\alpha}+\sum_{k=2}^{\infty}\left|a_{k}(\alpha)\right| z^{\alpha+k-1} \\
& +(-1)^{n} \sum_{k=1}^{\infty}\left|b_{k}(\alpha)\right| \overline{z^{\alpha+k-1}} \\
= & z^{\alpha}+\sum_{k=2}^{\infty} \frac{\alpha^{n}(1-\beta)(c)_{k-1}}{(\alpha+k-1)^{n}(a)_{k-1}} x_{k} z^{\alpha+k-1} \\
& +(-1)^{n} \sum_{k=1}^{\infty} \frac{\alpha^{n}(1-\beta)(c)_{k-1}}{(\alpha+k-1)^{n}(a)_{k-1}} y_{k} \overline{z^{\alpha+k-1}} \\
= & \sum_{k=2}^{\infty} h_{k}(z)^{\alpha} x_{k}+\sum_{k=1}^{\infty} g_{n k}(z)^{\alpha} y_{k} \\
& +z^{\alpha}\left(1-\sum_{k=2}^{\infty} x_{k}-\sum_{k=1}^{\infty} y_{k}\right) \\
= & \sum_{k=1}^{\infty}\left(h_{k}(z)^{\alpha} x_{k}+g_{n k}(z)^{\alpha} y_{k}\right),
\end{aligned}
$$

as required. 
Our next result is on distortion bounds for the functions in the class $V B_{n}^{\alpha} H(\beta, a, c)$.

Theorem 16. Let $f_{n}^{\alpha} \in V B_{n}^{\alpha} H(\beta, a, c)$. Then, for $|z|=r<1$, one has

$$
\begin{aligned}
\left|f_{n}(z)^{\alpha}\right| \leq & \left(1+\left|b_{1}(\alpha)\right|\right) r^{\alpha} \\
& +\left(\frac{\alpha}{\alpha+1}\right)^{n}\left(\frac{c(1-\beta)}{a}-\frac{1+\beta}{a}\left|b_{1}(\alpha)\right|\right) r^{\alpha+1}, \\
\left|f_{n}(z)^{\alpha}\right| \geq & \left(1-\left|b_{1}(\alpha)\right|\right) r^{\alpha} \\
& -\left(\frac{\alpha}{\alpha+1}\right)^{n}\left(\frac{c(1-\beta)}{a}-\frac{1+\beta}{a}\left|b_{1}(\alpha)\right|\right) r^{\alpha+1} .
\end{aligned}
$$

Proof. We only prove the right-hand inequality. The proof for the left-hand inequality is similar and will be omitted.

Let $f_{n}^{\alpha} \in V B_{n}^{\alpha} H(\beta, a, c)$. Taking the absolute value of $f_{n}^{\alpha}$, we obtain

$$
\begin{aligned}
\left|f_{n}(z)^{\alpha}\right| & \left|z^{\alpha}+\sum_{k=2}^{\infty} a_{k}(\alpha) z^{\alpha+k-1}+(-1)^{n} \sum_{k=1}^{\infty} b_{k}(\alpha) \overline{z^{\alpha+k-1}}\right| \\
\leq & \left(1+\left|b_{1}(\alpha)\right|\right) r^{\alpha}+\sum_{k=2}^{\infty}\left(\left|a_{k}(\alpha)\right|+\left|b_{k}(\alpha)\right|\right) r^{\alpha+k-1} \\
\leq & \left(1+\left|b_{1}(\alpha)\right|\right) r^{\alpha}+r^{\alpha+1} \sum_{k=2}^{\infty}\left(\left|a_{k}(\alpha)\right|+\left|b_{k}(\alpha)\right|\right) \\
\leq & \left(1+\left|b_{1}(\alpha)\right|\right) r^{\alpha}+\frac{\alpha^{n}(1-\beta) c}{(\alpha+1)^{n} a} \\
\leq & \times\left[\sum_{k=2}^{\infty} \frac{1}{1-\beta}\left(\frac{\alpha+1}{\alpha}\right)^{n} \frac{a}{c}\left|a_{k}(\alpha)\right|\right. \\
& \times\left[\frac{c(1-\beta)}{a}-\frac{a(1+\beta)}{a}\left|b_{1}(\alpha)\right|\right] r^{\alpha+1}, \\
\leq & \left(1+\left|b_{1}(\alpha)\right|\right) r^{\alpha}+\frac{\alpha^{n}(1-\beta)(c)}{(\alpha+1)^{n}(a)} \\
& \times\left[\frac{(a)_{k-1}}{(c)_{k-1}}\left(\frac{\alpha+k-1}{\alpha}\right)^{n}\left|b_{k}(\alpha)\right|\right] r_{k}(\alpha) \mid r^{\alpha+1} \\
& +\frac{(a)_{k-1}}{(c))_{k-1}}\left(\frac{\alpha+k-1}{\alpha+1}\right)^{n}\left|a_{k}(\alpha)\right|
\end{aligned}
$$

for $b_{1}(\alpha)<1$. This shows that the bound given in Theorem 16 is sharp.
The following covering results follow from the left-hand inequality in Theorem 18.

Corollary 17. If function $f_{n}^{\alpha}=h^{\alpha}+\overline{g^{\alpha}}$, where $h^{\alpha}$ and $g^{\alpha}$ are as given earlier in $V B_{n}^{\alpha} H(\beta, a, c)$, then

$$
\begin{aligned}
& \left\{\omega:|\omega| \leq\left(\frac{\alpha}{\alpha+1}\right)^{n}\left(\frac{\alpha+1}{\alpha}\right)^{n}\right. \\
& -\left(\frac{\alpha}{\alpha+1}\right)^{n} \frac{c}{a}(1-\beta)-\left(\frac{\alpha}{\alpha+1}\right)^{n}\left(\frac{\alpha+1}{\alpha}\right)^{n} \\
& \left.-\left(\frac{\alpha}{\alpha+1}\right)^{n} \frac{c}{a}(1-\beta)\left|b_{1}(\alpha)\right|\right\} \subset f_{n}(U) .
\end{aligned}
$$

For harmonic functions

$$
\begin{aligned}
& f_{n}(z)^{\alpha}=z^{\alpha}+\sum_{k=2}^{\infty}\left|a_{k}(\alpha)\right| z^{\alpha+k-1}+(-1)^{n} \sum_{k=1}^{\infty}\left|b_{k}(\alpha)\right| \overline{z^{\alpha+k-1}}, \\
& F_{n}(z)^{\alpha}=z^{\alpha}+\sum_{k=2}^{\infty}\left|A_{k}(\alpha)\right| z^{\alpha+k-1}+(-1)^{n} \sum_{k=1}^{\infty}\left|B_{k}(\alpha)\right| \overline{z^{\alpha+k-1}} .
\end{aligned}
$$

The convolution of $f_{n}^{\alpha}$ and $F_{n}^{\alpha}$ is given by

$$
\begin{aligned}
\left(f_{n}^{\alpha} * F_{n}^{\alpha}\right)(z)= & f_{n}(z)^{\alpha} * F_{n}^{\alpha}(z)^{\alpha} \\
= & z^{\alpha}+\left|a_{k}(\alpha)\right|\left|A_{k}(\alpha)\right| z^{\alpha+k-1} \\
& +(-1)^{n} \sum_{k=1}^{\infty}\left|b_{k}(\alpha)\right|\left|B_{k}(\alpha)\right| \overline{z^{\alpha+k-1}} .
\end{aligned}
$$

Using this definition, one shows that the class $V B_{n}^{\alpha} H(\beta, a, c)$ is closed under convolution.

Theorem 18. For $0 \leq \lambda \leq \beta<1$, let $f_{n}^{\alpha} \in V B_{n}^{\alpha} H(\beta, a, c)$, and let $F_{n}^{\alpha} \in V B_{n}^{\alpha} H(\beta, a, c)$. Then, $f_{n}^{\alpha} * F_{n}^{\alpha} \in V B_{n}^{\alpha} H(\beta, a, c) \subseteq$ $V B_{n}^{\alpha} H(\beta, a, c)$.

Proof. Let the functions

$$
\begin{aligned}
f_{n}(z)^{\alpha}= & z^{\alpha}+\sum_{k=2}^{\infty}\left|a_{k}(\alpha)\right| z^{\alpha+k-1} \\
& +(-1)^{n} \sum_{k=1}^{\infty}\left|b_{k}(\alpha)\right| \overline{z^{\alpha+k-1}} \text { be in } V B_{n}^{\alpha} H(\beta, a, c)
\end{aligned}
$$

and $F_{n}(z)^{\alpha}=z^{\alpha}+\sum_{k=2}^{\infty}\left|A_{k}(\alpha)\right| z^{\alpha+k-1}+(-1)^{n} \sum_{k=1}^{\infty} \mid B_{k}(\alpha) \overline{z^{\alpha+k-1}}$ be in $V B_{n}^{\alpha} H(\beta, a, c)$. Then, the convolution $f_{n}^{\alpha} * F_{n}^{\alpha}$ is given by $(89)$. 
We wish to show that the coefficients of $f_{n}^{\alpha} * F_{n}^{\alpha}$ satisfy the required condition given in Theorem 14. For $F_{n}^{\alpha} \in V B_{n}^{\alpha} H(\beta$, $a, c), A_{k}(\alpha) \leq 1$, and $\left|B_{k}(\alpha)\right| \leq 1$. Now, for the convolution function $\left(f_{n}^{\alpha} * F_{n}^{\alpha}\right)(z)$, we obtain

$$
\begin{aligned}
\sum_{k=2}^{\infty} \frac{1}{1-\beta} & \frac{(a)_{k-1}}{(c)_{k-1}}\left(\frac{\alpha+k-1}{\alpha}\right)^{n}\left|a_{k}(\alpha)\right|\left|A_{k}(\alpha)\right| \\
& +\sum_{k=1}^{\infty} \frac{1}{1-\beta} \frac{(a)_{k-1}}{(c)_{k-1}}\left(\frac{\alpha+k-1}{\alpha}\right)^{n}\left|b_{k}(\alpha)\right|\left|B_{k}(\alpha)\right| \\
\leq & \sum_{k=2}^{\infty} \frac{1}{1-\beta} \frac{(a)_{k-1}}{(c)_{k-1}}\left(\frac{\alpha+k-1}{\alpha}\right)^{n}\left|a_{k}(\alpha)\right| \\
& +\sum_{k=1}^{\infty} \frac{1}{1-\beta} \frac{(a)_{k-1}}{(c)_{k-1}}\left(\frac{\alpha+k-1}{\alpha}\right)^{n}\left|b_{k}(\alpha)\right|
\end{aligned}
$$

$\leq 1$.

Therefore $\left(f_{n}^{\alpha} * F_{n}^{\alpha}\right)(z) \in V B_{n}^{\alpha} H(\beta, a, c) \subseteq V B_{n}^{\alpha} H(\beta, a, c)$.

Next, we show that the class $V B_{n}^{\alpha} H(\beta, a, c)$ is closed under convex combinations of its members.

Let functions $f_{n j}(z)^{\alpha}$ be defined, for $j=1,2, \ldots$, by

$$
\begin{aligned}
& f_{n j}(z)^{\alpha} \\
& \quad=z^{\alpha}+\sum_{k=2}^{\infty}\left|a_{k, j}(\alpha)\right| z^{\alpha+k-1}+(-1)^{n} \sum_{k=1}^{\infty}\left|b_{k, j}(\alpha)\right| z^{\alpha+k-1} .
\end{aligned}
$$

Theorem 19. Let the functions $f_{n j}(z)^{\alpha}$ defined by (92) be in the class $V B_{n}^{\alpha} H(\beta, a, c)$ for every $j=1,2, \ldots, m$. Then, the functions $t_{j}(z)^{\alpha}$ defined by

$$
t_{j}(z)^{\alpha}=\sum_{j=1}^{m} \gamma_{j} f_{n j}(z) \quad\left(0 \leq \gamma_{j} \leq 1\right)
$$

are also in the class $V B_{n}^{\alpha} H(\beta, a, c)$, where $\sum_{j=1}^{m} \gamma_{j}=1$.

Proof. According to the definition of $t^{\alpha}$, we can write

$$
\begin{aligned}
t^{\alpha}= & z^{\alpha}+\sum_{k=2}^{\infty}\left(\sum_{j=1}^{m} \gamma_{j} a_{k, j}(\alpha)\right) z^{\alpha+k-1} \\
& +(-1)^{n} \sum_{k=1}^{\infty}\left(\sum_{j=1}^{m} \gamma_{j} a_{k, j}(\alpha)\right) z^{\alpha+k-1} .
\end{aligned}
$$

Furthermore, since $f_{n j}(z)^{\alpha}$ are in $V B_{n}^{\alpha} H(\beta, a, c)$ for every $(j=$ $1,2, \ldots$,$) , then by (66)$, we have

$$
\begin{aligned}
& \sum_{k=2}^{\infty} \frac{1}{1-\beta} \frac{(a)_{k-1}}{(c)_{k-1}}\left(\frac{\alpha+k-1}{\alpha}\right)^{n}\left(\sum_{j=1}^{m} \gamma_{j}\left|a_{k, j}(\alpha)\right|\right) \\
& \quad+\sum_{k=1}^{\infty} \frac{1}{1-\beta} \frac{(a)_{k-1}}{(c)_{k-1}}\left(\frac{\alpha+k-1}{\alpha}\right)^{n}\left(\sum_{j=1}^{m} \gamma_{j}\left|b_{k, j}(\alpha)\right|\right)
\end{aligned}
$$

$$
\begin{aligned}
& =\sum_{j=1}^{m} \gamma_{j}\left(\sum_{k=2}^{\infty} \frac{1}{1-\beta} \frac{(a)_{k-1}}{(c)_{k-1}}\left(\frac{\alpha+k-1}{\alpha}\right)^{n}\left|a_{k, j}(\alpha)\right|\right. \\
& \left.\quad+\sum_{k=1}^{\infty} \frac{1}{1-\beta} \frac{(a)_{k-1}}{(c)_{k-1}}\left(\frac{\alpha+k-1}{\alpha}\right)^{n}\left|b_{k, j}(\alpha)\right|\right) \\
& \leq \sum_{j=1}^{m} \gamma_{j}=1 .
\end{aligned}
$$

Hence, the theorem is proved.

Corollary 20. The class $V B_{n}^{\alpha} H(\beta, a, c)$ is closed under convex linear combination.

Proof. Let the functions $f_{n j}(z)^{\alpha}(j=1,2)$ defined by (92) be in the class $M V B_{n}^{\alpha} H(\beta, a, c)$. Then, the function $\Psi(z)^{\alpha}$ defined by

$$
\Psi(z)^{\alpha}=\mu f_{n 1}(z)^{\alpha}+(1-\mu) f_{n 2}(z)^{\alpha} \quad(0 \leq \mu \leq 1)
$$

is in the class $M V B_{n}^{\alpha} H(\lambda, a, c)$. Also, by taking $m=2, t_{1}=\mu$ and $t_{2}=(1-\mu)$ in Theorem 19, we have the above corollary.

With various special choices of the parameters involved, both the existing classes and new one could be derived.

\section{References}

[1] S. D. Bernard, "Convex and starlike univalent functions," Transactions of the American Mathematical Society, vol. 135, pp. 429446, 1969.

[2] M. Darus and R. W. Ibrahim, "Coefficient inequalities for a new class of univalent functions," Lobachevskii Journal of Mathematics, vol. 29, no. 4, pp. 221-229, 2008.

[3] B. A. Frasin, "Subclasses of analytic functions defined by Carlson-Shaffer linear operator," Tamsui Oxford Journal of Mathematical Sciences, vol. 23, no. 2, pp. 219-233, 2007.

[4] A. W. Goodman, Univalent Functions, vol. 1, Mariner Publishing, Tampa, Fla, USA, 1983.

[5] S. S. Miller and P. T. Mocanu, "Second order differential inequalities in the complex plane," Journal of Mathematical Analysis and Applications, vol. 65, no. 2, pp. 289-305, 1978.

[6] S. S. Miller and P. T. Mocanu, Differential Subordinations: Theory and Applications, vol. 225 of Pure and Applied Mathematics, Marcel Dekker, New York, NY, USA, 2000.

[7] H. Saitoh, "A linear operator and its applications of first order differential subordinations," Mathematica Japonica, vol. 44, no. 1, pp. 31-38, 1996.

[8] G. S. Sălăgean, "Subclasses of univalent functions," in Complex Analysis-Fifth Romanian-Finnish Seminar, vol. 1013 of Lecture Notes in Mathematics, pp. 362-372, Springer, New York, NY, USA, 1983.

[9] H. Mahzoon and S. Latha, "On certain properties of neighborhoods of multivalent functions involving the generalized saitoh operator," Journal of Inequalities in Pure and Applied Mathematics, vol. 10, no. 4, article 112, 6 pages, 2009.

[10] I. E. Bazilevič, "On a case of integrability in quadratures of the Loewner-Kufarev equation," Matematicheskii Sbornik, vol. 37, no. 79, pp. 471-476, 1955 (Russian). 
[11] R. Singh, "On Bazilevič functions," Proceedings of the American Mathematical Society, vol. 38, pp. 261-271, 1973.

[12] J. W. Noonan, “On close-to-convex functions of order $\beta$," Pacific Journal of Mathematics, vol. 44, no. 1, pp. 263-280, 1973.

[13] S. D. Bernardi, Bibliography of Schlicht Functions, Reprinted by Mariner Publishing, Tampa, Fla, USA, 1983, Courant Institute of Mathematical Sciences, New York University, 1996.

[14] S. Abdulhalim, "On a class of analytic functions involving the Salagean differential operator," Tamkang Journal of Mathematics, vol. 23, no. 1, pp. 51-58, 1992.

[15] T. H. MacGregor, "Functions whose derivative has a positive real part," Transactions of the American Mathematical Society, vol. 104, pp. 532-537, 1962.

[16] T. H. MacGregor, "The radius of convexity for starlike functions of order 1/2," Proceedings of the American Mathematical Society, vol. 14, no. 1, pp. 71-76, 1963.

[17] D. K. Thomas, "On Bazilevič functions," Transactions of the American Mathematical Society, vol. 132, no. 2, pp. 353-361, 1968.

[18] P. D. Tuan and V. V. Anh, "Radii of starlikeness and convexity for certain classes of analytic functions," Journal of Mathematical Analysis and Applications, vol. 64, no. 1, pp. 146-158, 1978.

[19] K. Yamaguchi, "On functions satisfying $R\{f(z) / z\}>0$," Proceedings of the American Mathematical Society, vol. 17, no. 3, pp. 588-591, 1966.

[20] T. O. Opoola, "On a new subclass of univalent functions," Mathematica, vol. 36, no. 2, tome (36), pp. 195-200, 1994.

[21] S. Singh, S. Gupta, and S. Singh, "An extension of the region of variability of a subclass of univalent functions," Journal of Inequalities in Pure and Applied Mathematics, vol. 10, no. 4, article 113, 7 pages, 2009.

[22] I. S. Jack, "Functions starlike and convex of order $\alpha$," Journal of the London Mathematical Society, vol. 3, pp. 469-474, 1971.

[23] H. Shiraishi and S. Owa, "Starlikeness and convexity for analytic functions concerned with Jack's lemma," International Journal of Open Problems in Computer Science and Mathematics, vol. 2, no. 1, pp. 37-47, 2009.

[24] J. M. Jahangiri, "Harmonic functions starlike in the unit disk," Journal of Mathematical Analysis and Applications, vol. 235, no. 2, pp. 470-477, 1999.

[25] J. M. Jahangiri, "Coefficient bounds and univalence criteria for harmonic functions with negative coefficients," Annales Universitatis Mariae Curie-Skłodowska A, vol. 52, no. 2, pp. 57-66, 1998.

[26] H. Silverman and E. M. Silvia, "Subclasses of harmonic univalent functions," New Zealand Journal of Mathematics, vol. 28, no. 2, pp. 275-284, 1999. 


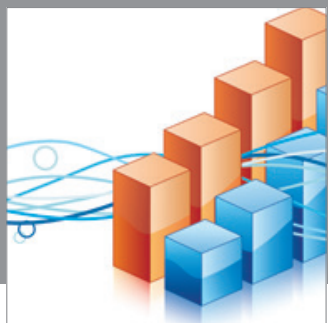

Advances in

Operations Research

mansans

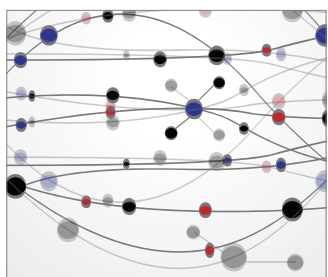

The Scientific World Journal
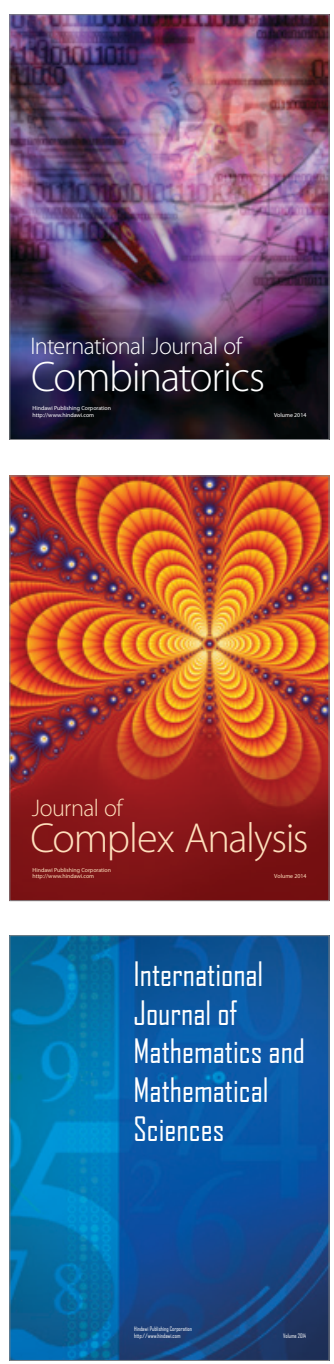
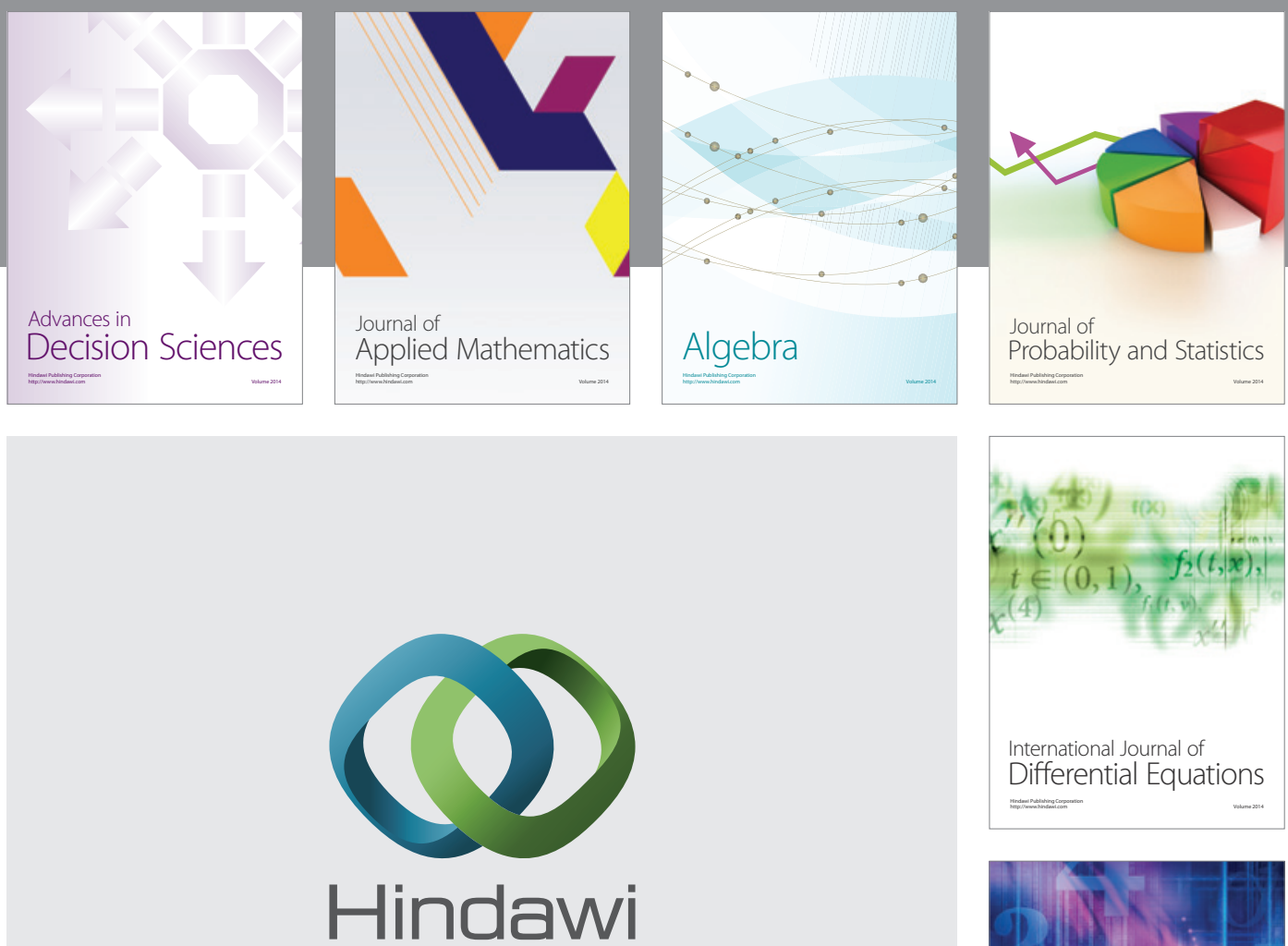

Submit your manuscripts at http://www.hindawi.com
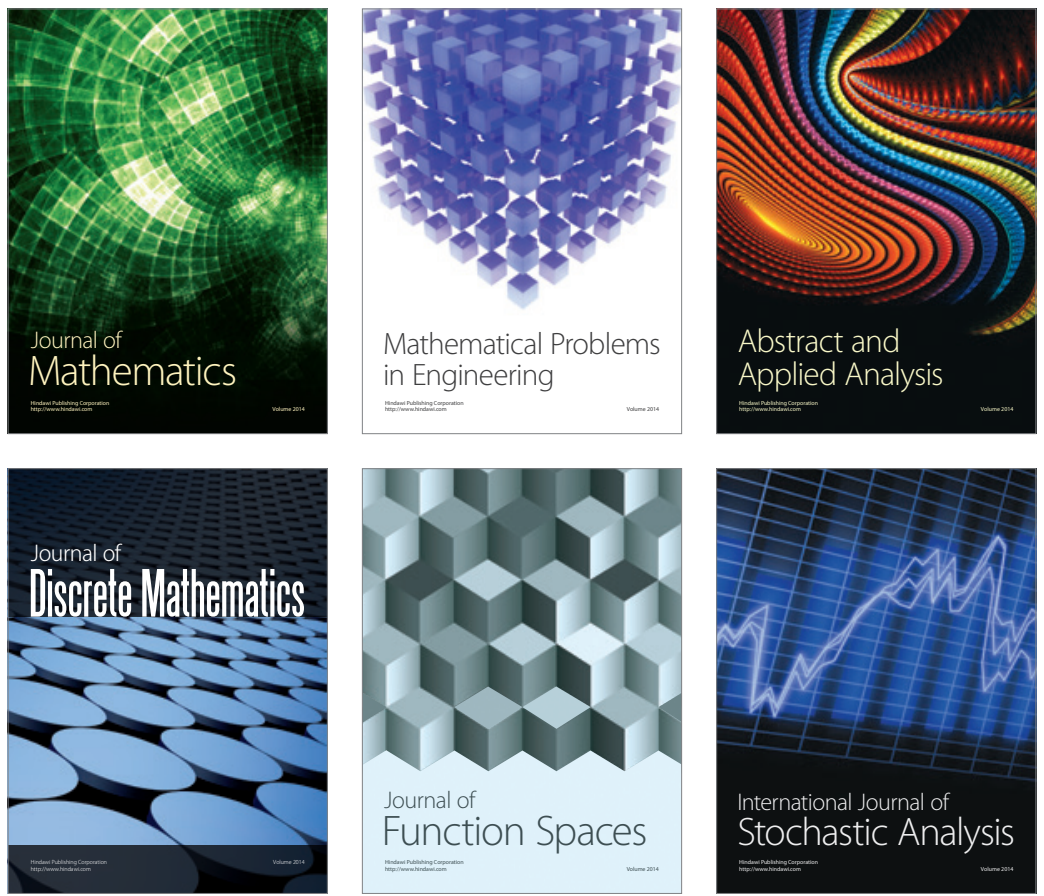

Journal of

Function Spaces

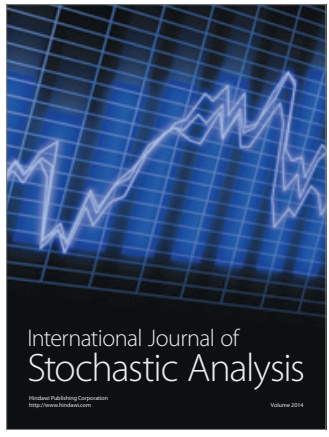

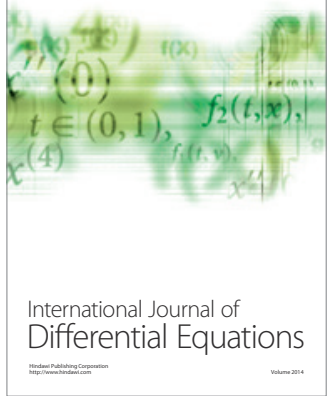
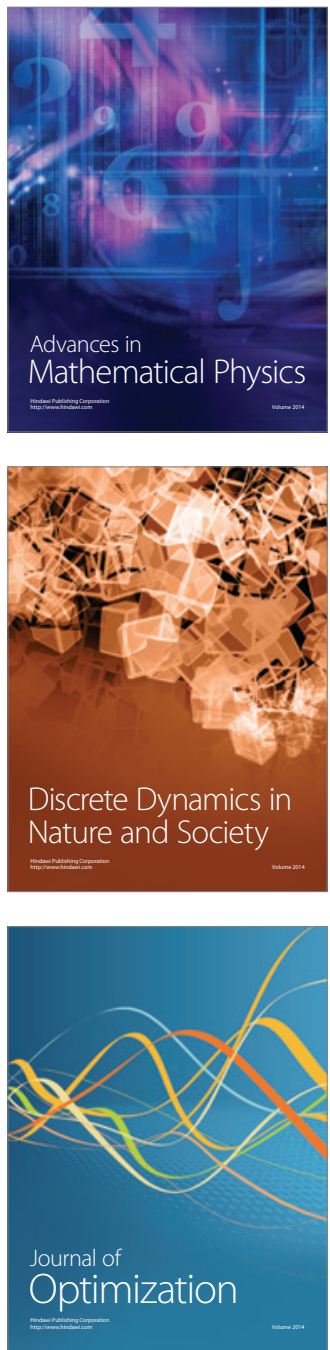